\title{
Chapter 1 \\ Advances in Wave Digital Modeling of Linear and Nonlinear Systems: A Summary
}

Alberto Bernardini

\begin{abstract}
This brief summarizes some of the main research results that I obtained during the three years, ranging from November 2015 to October 2018, as a Ph.D. student at Politecnico di Milano under the supervision of Professor Augusto Sarti, and that are contained in my doctoral dissertation, entitled "Advances in Wave Digital Modeling of Linear and Nonlinear Systems". The thesis provides contributions to all the main aspects of Wave Digital (WD) modeling of lumped systems: it introduces generalized definitions of wave variables; it presents novel WD models of one- and multi-port linear and nonlinear circuit elements; it discusses systematic techniques for the WD implementation of arbitrary connection networks and it describes a novel iterative method for the implementation of circuits with multiple nonlinear elements. Though WD methods usually focus on the discrete-time implementation of analog audio circuits; the methodologies addressed in the thesis are general enough as to be applicable to whatever system that can be described by an equivalent electric circuit.
\end{abstract}

\subsection{Introduction}

My doctoral dissertation presents various contributions to the recent evolution of modeling and implementation techniques of linear and nonlinear systems in the Wave Digital (WD) domain. The overarching goal of WD methods is to build digital implementations of analog systems, which are able to emulate the behavior of their analog counterpart in an efficient and accurate fashion. Though such methods usually focus on the WD modeling of analog audio circuits; the methodologies addressed in the thesis are general enough as to be applicable to whatever physical system that can be described by an equivalent electric circuit, which includes any system that can be thought of as a port-wise interconnection of lumped physical elements. The possibility of describing systems through electrical equivalents has relevant implications not only in the field of numerical simulation of physical phenomena, but also in the field of digital signal processing, as it allows us to model different

\footnotetext{
A. Bernardini $(\bowtie)$

Politecnico di Milano, Piazza Leonardo Da Vinci 32, 20133 Milano, Italy

e-mail: alberto.bernardini@polimi.it 
kinds of processing structures in a unified fashion and to easily manage the energetic properties of their input-output signals. However, digitally implementing nonlinear circuits in the Kirchhoff domain is not straightforward, because dual variables (currents and voltages) are related by implicit equations which make computability very hard. Mainstream Spice-like software, based on the Modified Nodal Analysis (MNA) framework [20], is not always suitable for realizing efficient and interactive digital applications, mainly because it requires the use of iterative methods based on large Jacobian matrices (e.g., Newton-Raphson) for solving multi-dimensional nonlinear systems of equations.

WD Filters (WDFs) are a very attractive alternative. During the seventies, Alfred Fettweis introduced WDFs as a special category of digital filters based on a lumped discretization of reference analog circuits [19]. A WDF is created by port-wise consideration of a reference circuit, i.e., decomposition into one-port and multi-port circuit elements, a linear transformation of Kirchhoff variables to wave signals (incident and reflected waves) with the introduction of a free parameter per port, called reference port resistance, and a discretization of reactive elements via the bilinear transform. Linear circuit elements, such as resistors, real sources, capacitors and inductors, can be described through wave mappings without instantaneous reflections, as they can be all "adapted" exploiting the mentioned free parameter; in such a way that local Delay-Free Loops (DFLs), i.e., implicit relations between wave variables are eliminated. Series and parallel topological connections between the elements are implemented using scattering topological junctions called "adaptors", which impose special adaptation conditions to eliminate global DFLs and ensure computability. It follows that WDFs, as opposed to approaches based on the MNA, allow us to model separately the topology and the elements of the reference circuit. Moreover, WDFs are characterized by stability, accuracy, pseudo-passivity, modularity and low computational complexity, making many real-time interactive applications easy to be realized. Most classical WD structures can be implemented in an explicit fashion, using binary connection trees, whose leaves are linear one-ports, nodes are 3-port adaptors and the root may be a nonlinear element. However, WDFs are also characterized by important limitations. The first main weakness of state-of-the-art WDFs is that WD structures, characterized by explicit input-output relations, can contain only one nonlinear element, as nonlinear elements cannot be adapted. In fact, the presence of multiple nonlinear elements might affect computability, which characterizes classical linear WDFs, as DFLs arise. As a second main limitation of traditional WDFs, up to three years ago, there were no systematic methods for modeling connection networks which embed non-reciprocal linear multi-ports, such as nullors or controlled sources. Finally, very few studies were presented on the use of discretization methods alternative to the bilinear transform and potentially adjustable step-size in WD structures.

The thesis presents various techniques to overcome the aforementioned limitations. After a review of the state of the art on WD methods up to 2015, the thesis is organized in five parts and each part presents original contributions to a specific important aspect of WD modeling. In the following, Sects. 1.2, 1.3, 1.4, 1.5 and 1.6 resume the content of Part I, Part II, Part III, Part IV and Part V of the thesis; each 
subsection providing a summary of a specific chapter. Section 1.7 concludes this brief and proposes some possible future developments.

\subsection{Part I: Rethinking Definitions of Waves}

Part I, containing Chaps. 2 and 3 of the thesis, discusses two families of definitions of wave signals; one based on one free parameter per port, the other based on two free parameters per port.

\subsubsection{Mono-Parametric Definitions of Wave Variables}

Chapter 2 presents a generalization of the traditional definitions of voltage waves, current waves and power-normalized waves, characterized by one port resistance per port. The generalization is done introducing a scalar parameter $\rho$ in expressions at the exponent of the port resistance. The generalized definition also includes novel definitions of waves never appeared in the literature. Such a generalized definition was firstly presented in [35] and it proved useful for modeling WD structures based on waves with different units of measure in an unified fashion. In fact, it was used in [30] and in [16] for modeling arbitrary reciprocal and non-reciprocal multi-port WD junctions. Chapter 2 also includes the scattering relations of some fundamental circuit elements and the scattering relations of series and parallel adaptors. It is shown that some scattering relations are invariant to the wave type (e.g., voltage waves, current waves, power-normalized waves), while other scattering relations are not invariant to the wave type since they depend on $\rho$.

\subsubsection{Biparametric Definitions of Wave Variables}

Chapter 3 presents the content of the published journal article [7], which introduces two dual definitions of power-normalized waves characterized by two free parameters per port, instead of one as it happens in traditional WDFs. It is shown that such dual definitions are two possible generalizations of the traditional definitions based on one port resistance and that when the two free parameters at the same port are set to be equal, they reduce to the traditional definition of waves. The WD structures based on the new definitions of wave variables are called Biparametric WDFs (BWDFs). It is shown that since BWDFs are characterized by more degrees of freedom than WDFs, they enable a higher modeling flexibility. For instance, all ports of adaptors based on biparametric definitions of waves can be made reflection free at the same time. This fact allows us to reduce the number of local DFLs in WD structures and to design adaptors whose behavior is uniform at all ports. Moreover, when the 
free parameters are properly set, series adaptors can be described using scattering matrices made of zeros in the diagonal entries and minus ones in the non-diagonal entries, while parallel adaptors can be described using scattering matrices made of zeros in the diagonal entries and ones in the non-diagonal entries. This further property is useful in many situations for reducing the number of multiplies required for implementing WD structures based on power-normalized waves. Finally, a discussion on the implementation of nonlinear circuits with multiple nonlinearities using BWDFs is provided. In particular, it is shown that, despite the use of adaptors with all reflection-free ports and less multipliers simplifies the modeling of nonlinear circuits with multiple nonlinearities in many aspects (e.g., considerably reducing the number of local DFLs and, consequently, the complexity of the implicit equations describing the circuit in the WD domain), not all global DFLs can be eliminated even using BWDFs and iterative methods are still required.

\subsection{Part II: Modeling Nonlinear One-Port and Multi-port Elements}

Part II, containing Chaps. 4, 5 and 6, is devoted to the modeling of nonlinear oneports and multi-ports in the WD domain. The common objective in all chapters is searching for the conditions that allow us to use explicit scattering relations for describing nonlinear one-ports and multi-ports in the WD domain. Also the use of the Newton-Raphson (NR) method for finding the solution of implicit scattering relations is discussed.

\subsubsection{Canonical Piecewise-Linear Representation of Curves in the Wave Digital Domain}

Chapter 4 presents the content of the published article [8], where a method is discussed that, starting from certain parameterized PieceWise-Linear (PWL) $i-v$ curves of one-ports in the Kirchhoff domain, expresses them in the WD domain using a global and explicit representation. Global, explicit representations of nonlinearities allow us to implement their input-output relations without managing look-up tables, performing data interpolation and/or using local iterative solvers. It is shown how certain curves (even some multi-valued functions in the Kirchhoff domain) can be represented as functions in explicit canonical PWL form in the WD domain. A general procedure is also provided that returns the conditions on the reference port resistance under which it is possible to find explicit mappings in the WD domain. 


\subsubsection{Wave Digital Modeling of Nonlinear Elements Using the Lambert Function}

Certain transcendental equations involving exponentials can be expressed in explicit form using the Lambert $W$ function. Chapter 5 presents the content of the published journal article [15], which explores how the $W$ function can be used to derive explicit WD models of some one-ports [32] and multi-ports characterized by exponential nonlinearities, such as banks of diodes in parallel and/or anti-parallel or BJTs in certain amplifier configurations.

\subsubsection{Wave Digital Modeling of Nonlinear 3-Terminal Devices for Virtual Analog Applications}

Chapter 6 presents the content of the article [11] submitted for publication and currently in peer review. It discusses an approach for modeling circuits containing arbitrary linear or nonlinear 3-terminal devices in the WD domain. Such an approach leads us to the definition of a general and flexible WD model for 3-terminal devices, whose number of ports ranges from 1 to 6 . The WD models of 3-terminal devices already discussed in the literature could be described as particular cases of the model presented in this chapter. As examples of applications of the proposed approach, WD models of the three most widespread types of transistors in audio circuitry, i.e., the MOSFET, the JFET and the BJT are developed. These WD models are designed to be used in Virtual Analog audio applications. It follows that the proposed models are derived with the aim of minimizing computational complexity, and avoiding implicit relations between port variables, as far as possible. Proposed MOSFET and JFET models result into third order equations to solve; therefore, closed-form wave scattering relations are obtained. Instead, the Ebers-Moll model describing the BJT is characterized by transcendental equations which cannot be solved in closed-form in the WD domain; consequently, iterative methods for finding their solutions have been studied. The standard NR method recently used in the literature on WDFs [25] do not satisfy all the requirements of robustness and efficiency needed in audio Virtual Analog applications. For this reason, a modified NR method is developed that exhibits a significantly higher robustness and convergence rate with respect to the traditional NR method, without compromising its efficiency. In particular, the behavior of the proposed modified NR method is far less sensitive to chosen initial guesses than the traditional NR method. The proposed method converges for any initial guess and any incident wave signal within reasonable ranges which are deduced from the parameters of the implemented reference circuits. 


\subsection{Part III: Modeling Connection Networks}

Part III, containing Chaps. 7, 8 and 9, focuses on the modeling of arbitrary connection networks in the WD domain. We refer to a connection network as a linear multi-port whose ports are connected to arbitrary loads, which might be single circuit elements or other networks. Connection networks in the WD domain are implemented using scattering junctions called adaptors. In turn, such WD junctions are characterized by scattering matrices, whose properties depend on the characteristics of the modeled reference connection network in the Kirchhoff domain and on the chosen definition of wave variables. Connection networks can be classified in two main classes; the class of reciprocal connection networks and the class of non-reciprocal connection networks. In turn, the class of reciprocal connection networks includes "wire connections" of arbitrary complexity (i.e., series/parallel connections or interconnections which are neither series nor parallel) and connection networks embedding reciprocal multi-ports, such as ideal two-winding or multi-winding transformers. On the other hand, non-reciprocal connection networks, embed one or more non-reciprocal multi-ports, such as nullors or controlled sources.

\subsubsection{Modeling Sallen-Key Audio Filters in the Wave Digital Domain}

In $[31,34]$ a method based on the MNA analysis of the reference connection network with instantaneous Thévenin equivalents connected to all its ports is presented. Ideal sources of instantaneous Thévenin equivalents are set equal to the waves incident to the WD junction and series resistances are set equal to reference port resistances (free parameters). Chapter 7 presents the content of the published article [29], where the MNA-based method is applied for implementing Sallen-Key filter circuits in the WD domain. In particular, the eighteen filter models presented by Sallen and Key in their historical 1955 manuscript [26] are grouped into nine classes, according to their topological properties. For each class the corresponding WD structure is derived.

\subsubsection{Modeling Circuits with Arbitrary Topologies and Active Linear Multiports Using Wave Digital Filters}

Chapter 8 presents the content of the published journal article [30], where the MNAbased method, presented in [31, 34] for modeling arbitrary connection networks using WD adaptors based on voltage waves, is extended in such a way that it can be applied to all mono-parametric definitions of waves using the generalized definition described in Chap. 2. Moreover, it is shown that, connecting instantaneous Norton equivalents to the ports of the connection network (instead of instantaneous 
Thévenin equivalents), in order to perform the MNA-based method, brings considerable advantages in terms of computational cost because the number of nodes is always reduced.

\subsubsection{Generalized Wave Digital Filter Realizations of Arbitrary Reciprocal Connection Networks}

Chapter 9 presents the content of the published journal article [16] in which the approach developed by Martens et al. [23] for modeling and efficiently implementing arbitrary reciprocal connection networks using WD scattering junctions based on voltage waves is extended to be used in WDFs based on the generalized monoparametric and biparametric definitions of waves discussed in Part I. The method presented in this chapter is less general than the one presented in Chap. 8 (and published in [30]), since it is limited to the modeling of reciprocal connection networks. However, as far as reciprocal connection networks are concerned, the computational efficiency of the proposed method generally surpasses or at least matches that of the method in [30], both when the cost of scattering and the sizes of linear systems to be inverted in order to form the scattering matrix are considered.

\subsection{Part IV: Implementation of Circuits with Multiple Nonlinearities}

As outlined in the introduction, circuits with one nonlinear element can be implemented using the trapezoidal discretization method obtaining explicit WD structures, i.e., digital structures without DFLs [24]. This is a considerable advantage of WD modeling over alternative Virtual Analog modeling approaches developed in the domain of voltages and currents, since they are typically characterized by implicit equations and require the use of iterative solvers. Unfortunately, such a great benefit is not preserved when circuits with multiple nonlinearities are considered because all DFLs cannot be eliminated [7]. However, even in those cases, working in the WD domain have proven to bring advantages. For instance, in [33] a method is introduced that allows us to group all the nonlinear elements "at the root" of the WD structure, enabling the possibility of separating the nonlinear part of the circuit from the linear one; then, the multivariate nonlinear system of equations describing the nonlinear part is solved using tabulation [13, 14, 33] or multivariate NR solvers [25]. Another approach is presented in [28], where multidimensional WDFs and the multivariate NR method are combined for solving nonlinear lumped circuits. An alternative approach, described in [27], exploits the contractivity property of certain WD structures for accommodating multiple nonlinearities using fixed point iteration schemes. However, from a theoretical stand point, in [27], contractivity ensuring 
convergence of the fixed point algorithm is proven and analyzed only considering linear WD structures.

Part IV, containing Chaps. 10, 11 and 12, is mainly devoted to the WD implementation of circuits with multiple nonlinearities using a further method called Scattering Iterative Method (SIM) that has been recently developed starting from the preliminary results presented in [6]. SIM is a relaxation method characterized by an iterative procedure that alternates a local scattering stage, devoted to the computation of waves reflected from each element, and a global scattering stage, devoted to the computation of waves reflected from a WD junction to which all elements are connected. Similarly to what happens in the implementation methods described in [27], wave signals circulate in the WD structure back and forth up to convergence. A proven theorem guarantees that SIM converges when applied to whichever circuit characterized by a reciprocal connection network and an arbitrary number of linear or nonlinear one-ports whose $i-v$ characteristic is monotonic increasing. It is worth noticing that the absence of guarantee of convergence when it comes to implement a nonlinear circuit using a whichever iterative method, does not necessarily imply that the method cannot be used for solving that circuit anyway; as a matter of fact, this happens in most situations in Spice-like software. The proven theorem not only helps in identifying a class of nonlinear circuits for which the convergence of SIM is theoretically ensured, but it also gives us insights about a strategy for increasing its convergence speed, properly setting the free parameters (port resistances). SIM is able to solve circuits with an arbitrary number $N_{\mathrm{nl}}$ of 2-terminal nonlinear elements using $N_{\mathrm{nl}}$ independent one-dimensional NR solvers instead of one $N_{\mathrm{nl}}$-dimensional NR solver. This fact implies a number of interesting features that greatly differentiate SIM from techniques based on high-dimensional NR solvers [25], like higher robustness (or even convergence guarantee, when dealing with elements with monotonically increasing $i-v$ curves), higher efficiency and the possibility of solving the nonlinearities in parallel threads of execution.

\subsubsection{Wave-Based Analysis of Large Nonlinear Photovoltaic Arrays}

Chapter 10 presents the content of the published journal article [4] in which SIM is employed for modeling and efficiently simulating large nonlinear photovoltaic (PV) arrays under partial shading conditions. Given the irradiation pattern and the nonlinear PV unit model (e.g., exponential junction model with bypass diode) with the corresponding parameters, the WD method rapidly computes the current-voltage curve at the load of the PV array [10]. The main features of the WD method are the use of a scattering matrix modeling the arbitrary PV array topology and the adoption of one-dimensional solvers to locally handle the nonlinear constitutive equations of $\mathrm{PV}$ units. A rigorous analysis of SIM shows that it can be considered as a fixed-point method that always converges to the PV array solution. Compared with standard 
Spice-like simulators, the WD method is up to 35 times faster for PV arrays made of thousands of units. This makes the proposed method palatable for the development of dedicated systems for the real time control and optimization of large PV plants, e.g, maximum power point trackers based on the rapid exploration of the $i-v$ curve at the load.

\subsubsection{Wave Digital Modeling of the Diode-Based Ring Modulator}

Chapter 11 presents the content of the published article [12] in which SIM is shown to be suitable also for the discrete-time emulation of audio circuits for Virtual Analog applications since it is robust and comparable to or more efficient than state-of-theart strategies in terms of computational cost. In particular, a WD model of a ring modulator circuit constituted of four diodes and two multi-winding transformers is derived. An implementation of the WD model based on SIM is then discussed and a proof of convergence is provided.

\subsubsection{Linear Multi-step Discretization Methods with Variable Step-Size in Nonlinear Wave Digital Structures}

Circuit modeling in the WD domain typically entails the implementation of capacitors and inductors employing the trapezoidal discretization method with fixed sampling step. However, in many cases, alternative discretization techniques, eventually based on adaptive sampling step, might be preferable. Chapter 12 presents the content of the journal article, in which an unified approach for implementing WD dynamic elements based on arbitrary linear multi-step discretization methods with variable step-size as time-varying WD Thévenin or Norton equivalents is discussed. Moreover, it is shown that such an approach is particularly suitable to be combined with SIM for solving circuits with multiple nonlinearities.

\subsection{Part V: New Applications of WD Principles}

Physical systems that do not come in the form of electrical circuits can often be accurately represented by electrical equivalents, and then modeled and implemented as WD structures. In some applications, it is even possible to define an electrical circuit that models a digital signal processing structure, and use its WD model to implement such system more efficiently. As an example of the sort, in Part V containing Chap. 13, it is shown how certain first-order beamforming systems can be represented using electrical equivalents and then implemented in the WD domain. 


\subsubsection{Wave Digital Implementation of Robust First-Order Differential Microphone Arrays}

As a secondary research project during my Ph.D., I worked on the modeling of Differential Microphone Arrays (DMAs) [2, 3, 17, 22]. In this regard, Chap. 13, presents the content of the published journal article [1], in which a novel time-domain WD implementation of robust first-order DMAs with uniform linear array geometry [36] is described. In particular, it is shown that the reference beamforming system, composed of an array of sensors and a bank of filters (one per sensor) designed in the frequency domain, can be exactly represented by a bank of simple electrical circuits. This fact allows us to derive a bank of WDFs, one per sensor, and obtain a time-domain realization of the same beamformer which is less computationally demanding than its counterpart implemented in the frequency domain. The proposed beamforming method is extremely efficient, as it requires at most two multipliers and one delay for each filter, where the necessary number of filters equals the number of physical microphones of the array, and it avoids the use of fractional delays. The update of the coefficients of the filters, required for reshaping the beampattern, has a significantly lower computational cost with respect to the time-domain methods presented in the literature [18]. This makes the proposed method suitable for real-time DMA applications with time-varying beampatterns.

\subsection{Conclusions and Future Works}

In this brief, I resumed the main contributions to WD modeling of lumped systems presented in my doctoral dissertation. As far as future work is concerned, I think the properties of BWDFs should be explored further. For instance, two parameters per port could be exploited for increasing the speed of convergence of SIM. Moreover, it is worth extending the applicability of SIM to circuits containing multi-port nonlinearities and nonreciprocal linear elements. Such extensions would pave the way towards the realization of new general purpose circuit simulators that are potentially more efficient, robust and parallelizable than mainstream Spice-like software.

Another promising application of the presented WD modeling techniques is the design of inverse nonlinear systems, given direct systems represented as electrical equivalent circuits. In fact, the inverse of a nonlinear circuit system can be obtained by properly adding a nullor to the direct system itself [21]. In this regard, preliminary results published in the conference paper [9] show how the approach for modeling non-reciproca junctions in the WD domain, presented in [30], can be exploited for modeling the inverse of certain single-input-single-output audio circuits. 


\section{References}

1. Bernardini A, Antonacci F, Sarti A (2018) Wave digital implementation of robust first-order differential microphone arrays. IEEE Signal Process Lett 25(2):253-257. https://doi.org/10. 1109/LSP.2017.2787484

2. Bernardini A, D'Aria M, Sannino R (2017) Beamforming method based on arrays of microphones and corresponding apparatus. US Patent US9913030B2

3. Bernardini A, D'Aria M, Sannino R, Sarti A (2017) Efficient continuous beam steering for planar arrays of differential microphones. IEEE Signal Process Lett 24(6):794-798. https:// doi.org/10.1109/LSP.2017.2695082

4. Bernardini A, Maffezzoni P, Daniel L, Sarti A (2018) Wave-based analysis of large nonlinear photovoltaic arrays. IEEE Trans Circuits Syst I Regul Pap 65(4):1363-1376. https://doi.org/ 10.1109/TCSI.2017.2756917

5. Bernardini A, Maffezzoni P, Sarti A (2019) Linear multi-step discretization methods with variable step-size in nonlinear wave digital structures for virtual analog modeling. IEEE/ACM Trans Audio Speech Language Process 27(11):1763-1776. https://doi.org/10.1109/TASLP. 2019.2931759

6. Bernardini A, Sarti A (2016) Dynamic adaptation of instantaneous nonlinear bipoles in wave digital networks. In: Proceedings of the 24th European signal processing conference Budapest, Hungary. https://doi.org/10.1109/EUSIPCO.2016.7760406

7. Bernardini A, Sarti A (2017) Biparametric wave digital filters. IEEE Trans Circuits Syst I Regul Pap 64(7):1826-1838. https://doi.org/10.1109/TCSI.2017.2679007

8. Bernardini A, Sarti A (2017) Canonical piecewise-linear representation of curves in the wave digital domain. In: Proceedings of the 25th European signal processing conference (EUSIPCO), pp 1125-1129. Kos, Greece. https://doi.org/10.23919/EUSIPCO.2017.8081383

9. Bernardini A, Sarti A (2019) Towards inverse virtual analog modeling. In: Proceedings of the 22nd conference digital audio effects, Birmingham, UK. Paper number \#8

10. Bernardini A, Sarti A, Maffezzoni P, Daniel L (2018) Wave digital-based variability analysis of electrical mismatch in photovoltaic arrays. In: Proceedings of the IEEE international symposium on circuits and systems (ISCAS), pp 1-5. Florence, Italy. https://doi.org/10.1109/ISCAS. 2018.8351026

11. Bernardini A, Vergani AE, Sarti A (2019) Wave digital modeling of nonlinear 3-terminal devices for virtual analog applications. Springer circuits, systems, and signal processing (CSSP) (Submitted)

12. Bernardini A, Werner KJ, Maffezzoni P, Sarti A (2018) Wave digital modeling of the diodebased ring modulator. In: Proceedings of the 144th convention audio engineering society, Milan, Italy. Convention paper \#10015

13. Bernardini A, Werner KJ, Sarti A, Smith III JO (2015) Modeling a class of multi-port nonlinearities in wave digital structures. In: Proceedings of the European signal processing conference, Nice, France. https://doi.org/10.1109/EUSIPCO.2015.7362466

14. Bernardini A, Werner KJ, Sarti A, Smith III JO (2015) Multi-port nonlinearities in wave digital structures. In: Proceedings of the IEEE International Symposium on Signals, Circuits and Systems, Iaşi, Romania. https://doi.org/10.1109/ISSCS.2015.7203989

15. Bernardini A, Werner KJ, Sarti A, Smith III JO (2016) Modeling nonlinear wave digital elements using the Lambert function. IEEE Trans Circuits Syst I Regul Pap 63(8):1231-1242. https://doi.org/10.1109/TCSI.2016.2573119

16. Bernardini A, Werner KJ, Smith III JO, Sarti A (2019) Generalized wave digital filter realizations of arbitrary reciprocal connection networks. IEEE Trans Circuits Syst I Regul Pap 66(2):694-707. https://doi.org/10.1109/TCSI.2018.2867508

17. Borra F, Bernardini A, Antonacci F, Sarti A (2019) Uniform linear arrays of first-order steerable differential microphones. IEEE/ACM Trans Audio Speech Language Process 27(12):19061918. https://doi.org/10.1109/TASLP.2019.2934567 
18. Buchris Y, Cohen I, Benesty J (2016) First-order differential microphone arrays from a timedomain broadband perspective. In: 2016 IEEE international workshop on acoustic signal enhancement (IWAENC), pp 1-5. https://doi.org/10.1109/IWAENC.2016.7602886

19. Fettweis A (1986) Wave digital filters: theory and practice. Proc IEEE 74(2):270-327. https:// doi.org/10.1109/PROC.1986.13458

20. Ho CW, Ruehli AE, Brennan PA (1975) The modified nodal approach to network analysis. IEEE Trans Circuits Syst 22(6):504-509. https://doi.org/10.1109/TCS.1975.1084079

21. Leuciuc A (1998) The realization of inverse system for circuits containing nullors with applications in chaos synchronization. Int J Circuit Theory Appl 26(1):1-12. https://doi.org/10. 1002/(SICI) 1097-007X(199801/02)26:1<1::AID-CTA989>3.0.CO;2-B

22. Lovatello J, Bernardini A, Sarti A (2018) Steerable circular differential microphone arrays. In: 26th European signal processing conference (EUSIPCO), pp 11-15. Rome, Italy. https://doi. org/10.23919/EUSIPCO.2018.8553083

23. Martens GO, Meerkötter K (1976) On N-port adaptors for wave digital filters with application to a bridged-tee filter. In: Proceedings of the IEEE international symposium on circuits and systems, pp. 514-517. Munich, Germany

24. Meerkötter K, Scholz R (1989) Digital simulation of nonlinear circuits by wave digital filter principles. In: IEEE international symposium on circuits and systems, pp 720-723. https://doi. org/10.1109/ISCAS.1989.100452

25. Olsen MJ, Werner KJ, Smith III JO (2016) Resolving grouped nonlinearities in wave digital filters using iterative techniques. In: Proceedings 19th international conference digital audio effects, pp 279-286. Brno, Czech Republic

26. Sallen RP, Key EL (1955) A practical method of designing RC active filters. IRE Trans Circuit Theory 2(1):74-85. https://doi.org/10.1109/TCT.1955.6500159

27. Schwerdtfeger T, Kummert A (2014) A multidimensional approach to Wave Digital Filters with multiple nonlinearities. In: 22nd Proceedings of the European signal processing conference (EUSIPCO), pp 2405-2409. Lisbon, Portugal

28. Schwerdtfeger T, Kummert A (2015) Newton's method for modularity-preserving multidimensional wave digital filters. In: Proceedings of the IEEE international workshop multidimensional system Vila Real, Portugal

29. Verasani M, Bernardini A, Sarti A (2017) Modeling sallen-key audio filters in the wave digital domain. In: 2017 IEEE international conference on acoustics, speech and signal processing (ICASSP), pp 431-435. New Orleans, LA. https://doi.org/10.1109/ICASSP.2017.7952192

30. Werner KJ, Bernardini A, Smith III JO, Sarti A (2018) Modeling circuits with arbitrary topologies and active linear multiports using wave digital filters. IEEE Trans Circuits Syst I Regul Pap 65(12):4233-4246. https://doi.org/10.1109/TCSI.2018.2837912

31. Werner KJ, Dunkel WR, Rest M, Olsen MJ, Smith III JO (2016) Wave digital filter modeling of circuits with operational amplifiers. In: Proceedings of the european signal processing conference, pp 1033-1037. Budapest, Hungary. https://doi.org/10.1109/EUSIPCO.2016.7760405

32. Werner KJ, Nangia V, Bernardini A, Smith III JO, Sarti A (2015) An improved and generalized diode clipper model for wave digital filters. In: Proceedings of the 139th convention audio engineering society, New York. Convention paper. \#9360

33. Werner KJ, Nangia V, Smith III JO, Abel JS (2015) Resolving wave digital filters with multiple/multiport nonlinearities. In: Proceedings of the 18th international conference on digital audio effects, pp 387-394. Trondheim, Norway

34. Werner KJ, Smith III JO, Abel JS (2015) Wave digital filter adaptors for arbitrary topologies and multiport linear elements. In: Proceedings of the 18th international conference on digital audio effects, pp 379-386. Trondheim, Norway

35. Werner KJ (2016) Virtual analog modeling of audio circuitry using wave digital filters. $\mathrm{PhD}$ Dissertation, Stanford University, CA

36. Zhao L, Benesty J, Chen J (2014) Design of robust differential microphone arrays. IEEE/ACM Trans Audio Speech Lang Process 22(10):1455-1466. https://doi.org/10.1109/TASLP.2014. 2337844 
Open Access This chapter is licensed under the terms of the Creative Commons Attribution 4.0 International License (http://creativecommons.org/licenses/by/4.0/), which permits use, sharing, adaptation, distribution and reproduction in any medium or format, as long as you give appropriate credit to the original author(s) and the source, provide a link to the Creative Commons license and indicate if changes were made.

The images or other third party material in this chapter are included in the chapter's Creative Commons license, unless indicated otherwise in a credit line to the material. If material is not included in the chapter's Creative Commons license and your intended use is not permitted by statutory regulation or exceeds the permitted use, you will need to obtain permission directly from the copyright holder. 